EPJ Web of Conferences 108, 02005 (2016)

DOI: 10.1051/epjconf/201610802005

(C) Owned by the authors, published by EDP Sciences, 2016

\title{
Numerical Calculation of Scaling Exponents of Percolation Process in the Framework of Renormalization Group Approach
}

\author{
L. Ts. Adzhemyan ${ }^{1}$, M. Hnatič2,3,4,a , M. Kompaniets ${ }^{1}$, T. Lučivjanský' ${ }^{2,5}$, and L. Mižišin²,4,b \\ ${ }^{1}$ Department of Theoretical Physics, St. Petersburg University, Ulyanovskaya 1, \\ 198504 St. Petersburg-Petrodvorets, Russia \\ ${ }^{2}$ Department of Theoretical Physics, SAS, Institute of Experimental Physics, Watsonova 47, \\ 04001 Košice, Slovakia \\ ${ }^{3}$ Bogoliubov Laboratory of Theoretical Physics, Joint Institute for Nuclear Research, Joliot-Curie 6, \\ 141980 Dubna, Moscow Region, Russia \\ ${ }^{4}$ Faculty of Science, P. J. Šafárik University, Šrobárova 2, 04154 Košice, Slovakia \\ ${ }^{5}$ Fakultät für Physik, Universität Duisburg-Essen, D-47048 Duisburg, Germany
}

\begin{abstract}
The renormalization group theory is used to the study of the directed bond percolation (Gribov process) near its second-order phase transition between absorbing and active state. We present a numerical calculation of the renormalization group functions in the $\epsilon$-expansion where $\epsilon$ is the deviation from the upper critical dimension $d_{c}=4$. Within this procedure anomalous dimensions $\gamma$ are expressed in terms of irreducible renormalized Feynman diagrams and thus the calculation of renormalization constants could be entirely skipped. The renormalization group is included by means of the $R$ operation, and for computational purposes we choose the null momentum subtraction scheme.
\end{abstract}

\section{Introduction}

The renormalization group (RG) method is a especially suitable for studying various critical phenomena $[1,2]$. From a computational point of view it provides techniques for the perturbative calculation of different critical exponents. One of the most prominent dynamical models [3] which exhibits a second order phase transition is the directed bond percolation $[4,5]$.

In the physical literature it is known also as the Schlögl first reaction [6]. Among others it explains hadron interactions at very high energies (Reggeon field theory) [7], stochastic reaction-diffusion processes on a lattice [8], spreading of infection diseases [9], etc. The critical exponents are calculable in the form of perturbative expansion in a formally small parameter $\epsilon$. We note that two-loop results for the exponents $z$ and $\delta$ were obtained in [10] and exponents $v$ and $\beta$ later on in [11]. All necessary information concerning the percolation process in terms of the reaction-diffusion model can be found in the review article [9].

As discussed in detail in the literature [1] (Part 3.5) or [2] (Part 7.5) the central idea behind the renormalization group is the freedom to choose the particular renormalization scheme. This results in

\footnotetext{
ae-mail: hnatic@saske.sk

be-mail: lukas.mizisin@student.upjs.sk
} 
a theory which is finite with respect to ultraviolet divergences and regarding universal quantities they lead to the same result. For practical numerical calculations it is convenient to choose the subtractions at the normalization point $\left\{p=0, \omega=0, \tau=\mu^{2}\right\}$ as explained in [1] (see Eq. (3.18)).

Furthermore, in the renormalized Green function it is possible to replace the additional contribution of the renormalized constant by the operator $R[1]$ applied to Green functions

$$
\Gamma^{R}=R \Gamma=(1-K) R^{\prime} \Gamma,
$$

where $R^{\prime}$ is the incomplete operator $R$ that cancels the divergences in the subgraphs of a given graph and the operator $(1-K)$ eliminates the remaining superficial divergence.

The main part of this work is to show the basic steps of the alghoritmic procedure, which allow us to reproduce the known two-loop results to very high precision. Moreover it is easy to generalize our procedure to high orders and thus obtain more reliable results.

\section{Renormalization of the model}

A field theoretical formulation of the percolation process [9] is based on the action

$$
S=\psi^{\dagger}\left(-\partial_{t}+D_{0} \partial^{2}-D_{0} \tau_{0}\right) \psi+\frac{D_{0} \lambda_{0}}{2}\left[\left(\psi^{\dagger}\right)^{2} \psi-\psi^{\dagger} \psi^{2}\right],
$$

where $\psi$ is the coarse-grain density of the percolating agents, $\psi^{\dagger}$ is an auxiliary (Martin-Siggia-Rose) response field, $D_{0}$ is the diffusion constant, $\lambda_{0}$ is a positive coupling constant and $\tau_{0}$ is the deviation from the threshold value of the injected probability (an analog of critical temperature in static models). The model is studied near its critical dimension $\epsilon=4-d$ in the region where $\tau_{0}$ acquires its critical value. The expansion parameter of the perturbation theory is $\lambda_{0}^{2}$ rather than $\lambda_{0}$ as it could be easily seen by a direct inspection of the Feynman diagrams. Hence it is more convenient to introduce a new charge $u=\lambda^{2}$. The renormalized action functional can be written in the following form

$$
S_{R}=\psi^{\dagger}\left(-Z_{1} \partial_{t}+Z_{2} D \partial^{2}-Z_{3} D \tau\right) \psi+\frac{Z_{4} D \lambda \mu^{\epsilon}}{2}\left[\left(\psi^{\dagger}\right)^{2} \psi-\psi^{\dagger} \psi^{2}\right] .
$$

It can be shown [9] that this kind of model is multiplicatively renormalizable. Furthermore the action functional $S_{R}$ can also be obtained from the action $S$ by the standard procedure of multiplicative renormalization of all the fields and parameters

$$
\psi_{0}=\psi Z_{\psi}, \quad \psi_{0}^{\dagger}=\psi^{\dagger} Z_{\psi^{\dagger}}, \quad D_{0}=D Z_{D}, \quad \lambda_{0}=\lambda \mu^{\epsilon} Z_{\lambda}, \quad \tau_{0}=\tau Z_{\tau} .
$$

The relations between the renormalized constants $Z_{i}, i=1,2,3,4$ are obtained in a straightforward fashion and read

$$
\begin{array}{ll}
Z_{1}=Z_{\psi} Z_{\psi^{\dagger}}, & Z_{2}=Z_{D} Z_{\psi} Z_{\psi^{\dagger}}, \\
Z_{3}=Z_{D} Z_{\tau} Z_{\psi} Z_{\psi^{\dagger}}, & Z_{4}=Z_{D} Z_{\lambda} Z_{\psi^{\dagger}}^{2} Z_{\psi}=Z_{D} Z_{\lambda} Z_{\psi^{\dagger}} Z_{\psi^{2}}^{2} .
\end{array}
$$

Moreover, the relation $Z_{\psi}=Z_{\psi^{\dagger}}$ is satisfied. In this work, at the normalization point (NP), $p=0$, $\omega=0$ and $\tau=\mu^{2}$ is considered. The counterterms are then specified at the normalization point, and it is advantageous to express the renormalization constants in terms of normalized Green functions

$$
\begin{array}{ll}
\bar{\Gamma}_{1}=\left.\partial_{i \omega} \Gamma_{\psi^{\dagger} \psi}\right|_{p=0, \omega=0}, & \bar{\Gamma}_{3}=-\left.\frac{\Gamma_{\psi^{\dagger} \psi}-\left.\Gamma_{\psi^{\dagger} \psi}\right|_{\tau=0}}{D \tau}\right|_{p=0, \omega=0}, \\
\bar{\Gamma}_{2}=-\left.\frac{1}{2 D} \partial_{p}^{2} \Gamma_{\psi^{\dagger} \psi}\right|_{p=0, \omega=0}, & \bar{\Gamma}_{4}=\left.\frac{\Gamma_{\psi^{\dagger} \psi^{\dagger} \psi}-\Gamma_{\psi^{\dagger} \psi \psi}}{D \lambda \mu^{\epsilon}}\right|_{p=0, \omega=0},
\end{array}
$$


that satisfy the following conditions $\left.\bar{\Gamma}_{i}^{R}\right|_{\tau=\mu^{2}}=1, \quad i=1,2,3,4$. The RG constants defined by these conditions do not depend on $\tau$, like in the minimal subtraction (MS) scheme (see, e.g., Parts 3.3-3.5 in [1]). Accordingly, the RG equations are the same as in the MS scheme

$$
\left(\mu \partial_{\mu}+\beta_{u} \partial_{u}-\tau \gamma_{\tau} \partial_{\tau}-D \gamma_{D} \partial_{D}\right) \Gamma_{i}^{R}=\left(n_{\psi} \gamma_{\psi}+n_{\psi^{\dagger}} \gamma_{\psi^{\dagger}}\right) \Gamma_{i}^{R},
$$

where $\mu$ is a reference mass scale, $n_{\psi}$ and $n_{\psi_{\dagger}}$ are the numbers of the corresponding fields entering the Green function under consideration, $\gamma_{x}=\mu \partial_{\mu} \log Z_{x}$ are anomalous dimensions and $\beta_{u}=u\left(-2 \epsilon-\gamma_{u}\right)$ is a beta function describing the flow of the charge $u$ under the RG transformation [1]. Using these equations we find relations for the normalized functions

$$
\left(\mu \partial_{\mu}+\beta_{u} \partial_{u}-\tau \gamma_{\tau} \partial_{\tau}-D \gamma_{D} \partial_{D}\right) \bar{\Gamma}_{i}^{R}=\gamma_{i} \bar{\Gamma}_{i}^{R}
$$

Here, anomalous dimensions $\gamma_{i}$ are obtained from the relations (5)

$$
\gamma_{1}=2 \gamma_{\psi}, \quad \gamma_{3}=2 \gamma_{\psi}+\gamma_{D}+\gamma_{\tau}, \quad \gamma_{2}=2 \gamma_{\psi}+\gamma_{D}, \quad \gamma_{4}=3 \gamma_{\psi}+\gamma_{D}+\gamma_{\lambda} .
$$

Taking into account the renormalization scheme, the anomalous dimension can be expressed in terms of the renormalized derivatives of the one-particle irreducible Green function $\bar{\Gamma}_{i}$ at the normalization point [12-14]

$$
F_{i} \equiv-\left.\left[\tilde{\tau} \partial_{\tilde{\tau}} \bar{\Gamma}_{i}^{R}(\tilde{\tau})\right]\right|_{\tilde{\tau}=1}, \quad i=1,2,4,
$$

where $\tilde{\tau}=\tau / \mu^{2}$. At the normalization point $(\tilde{\tau}=1), \gamma_{i}$ takes the form $[12,13]$

$$
\gamma_{i}=\frac{2 F_{i}}{1+F_{2}-F_{3}}, \quad i=1,2,4 .
$$

For later considerations it is reasonable to introduce the new functions (see [13])

$$
\left.f_{i} \equiv R\left[-\tilde{\tau} \partial_{\tilde{\tau}} \bar{\Gamma}_{i}(\tilde{\tau})\right]\right|_{\tilde{\tau}=1} .
$$

These functions are related to the functions $F_{i}$ of (10) by

$$
f_{i}-F_{i}=f_{i} F_{3}, \quad i=1,2,4 .
$$

We rewrite equations in (11) to obtain relations for anomalous dimensions in terms of the renormalized derivatives of the one-irreducible Green function $\bar{\Gamma}_{i}$ with respect to $\tilde{\tau}$ at the normalized point

$$
\gamma_{i}=\frac{2 f_{i}}{1+f_{2}}, \quad i=1,2,4 .
$$

The main benefit of the procedure based on (12) is that the operator $R$ is taken at the normalization point and it can be expressed in terms of a subtracting operator $1-K_{i}$ that eliminates all divergences from the Feynman graphs [1]

$$
R \Gamma=\prod_{i}\left(1-K_{i}\right) \Gamma
$$

where the product is taken over all relevant subgraphs of the given Feynman graph, including also the graph as a whole. In the NM scheme we obtain the following representation for the $R$-operator $[12,14]$

$$
R \chi=\prod_{i} \frac{1}{n_{i} !} \int_{0}^{1} d a_{i}\left(1-a_{i}\right)^{n_{i}} \partial_{a_{i}}^{n_{i}+1} \chi(\{a\}),
$$


where the product is taken over all one-irreducible subgraphs $\chi_{i}$ (again including the graph $\chi$ as a whole) with the canonical dimension $n_{i} \geq 0$ and $a_{i}$ is a parameter that stretches momenta flowing into the $i$-th subgraph inside this graph. The main outcome of this approach is that the integrals are finite for $\epsilon=0$. This scheme enables us to calculate a contribution from each diagram to counterterms [12]

$$
Z_{i}=\frac{2}{n \epsilon}\left[f_{i}-J \bar{\Gamma}_{i}^{(n)}\right],
$$

where $n$ is a number of loops. The second term on the right hand side stands for a sum of diagrams of lower order perturbation theory. This allows us to recursively calculate counterterms in the NM scheme at the normalized point and thus gives us an opportunity to compare the results with ones obtained in the MS scheme.

\section{Calculation of anomalous dimensions}

In this part, we illustrate the method described in the previous section by its application to a specific diagram. Let us consider a two-loop contribution to $f_{3}$ from (12) determined by the two-loop threepoint diagram of $\Gamma_{4}$ with the dimension $n_{\chi}=0$. The diagram has one relevant subgraph: the subgraph

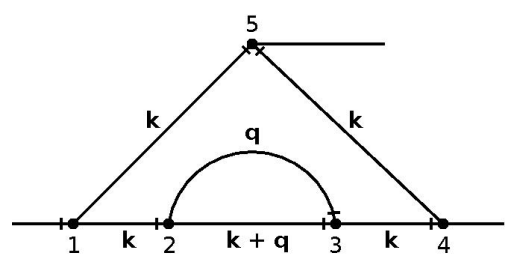

Figure 1. The two-loop Feynman diagram of $\Gamma_{4}$ with the symmetry factor equal to one

given by the vertices $\{2,3\}$ with the dimension $n_{a}=2$.

The action of the differential operator $-\tilde{\tau} \partial_{\tilde{\tau}}$ on the line $G(k)=1 /\left(k^{2}+\tilde{\tau}\right)$ produces an additional factor $1 /\left(k^{2}+\tilde{\tau}\right)$ and it corresponds to the insertion of a unit vertex into the propagator line. Graphically it will be denoted by an additional two-point interaction vertex. The application of the operator to the diagram results into a sum over all possible insertions of the vertex

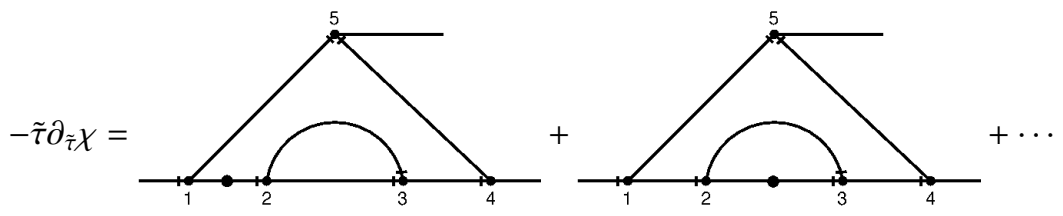

The next step consists of the inclusion of the operator $R$, but the analysis of each diagram has to be made separately. For example for the first diagram on the right hand side of (18), with the point outside the subgraph, the dimension of the subgraph remains unchanged and $n_{a}=2$ remains valid. On the other hand, in the second graph, the subgraph becomes logarithmic and the corresponding dimension is changed to $n_{a}=0$. In this way we obtain the expansion

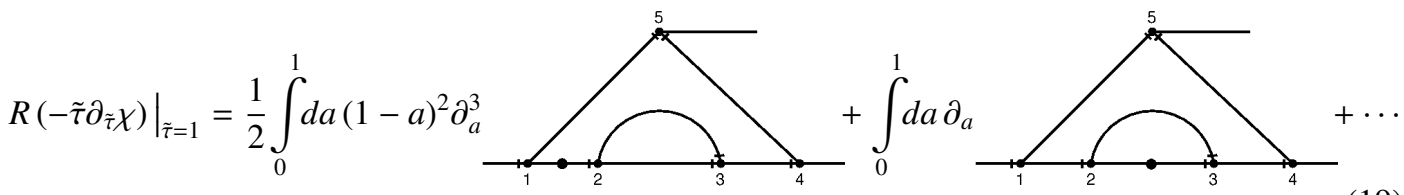


Further it is necessary to multiply all external parameters for a given subgraph by the parameter $a$. For the propatagor line this means that $G(q+k)=1 /\left[(a q+k)^{2}+1\right]$.

Combining (9) and (12) we can derive relations for the anomalous dimensions $\gamma$ for the fields and the parameters of the model,

$$
\gamma_{\psi}=\frac{f_{1}}{1+f_{2}}, \quad \gamma_{D}=\frac{2\left(f_{2}-f_{1}\right)}{1+f_{2}}, \quad \gamma_{u}=2 \frac{2 f_{4}-f_{1}-2 f_{2}}{1+f_{2}},
$$

where $f_{i}$ s are given up to the two-loop approximation by the following expressions:

$$
f_{1}=-\frac{u}{16}+\frac{u \epsilon}{32}+0.0152772 u^{2}, \quad f_{2}=-\frac{u}{32}+\frac{u \epsilon}{64}+0.0062804 u^{2}, \quad f_{4}=-\frac{u}{4}+\frac{u \epsilon}{8}+0.117185 u^{2} .
$$

These results were obtained by a numerical calculation in which the actual form of integrals is determined by the $R$ operator using the Feynman representation. Subsequently, the momentum integrals are calculated by Monte Carlo methods [15]. To the second order of perturbation theory, there are two diagrams for the function $\Gamma_{\psi^{+} \psi}$ and 11 diagrams for the function $\Gamma_{\psi^{\dagger} \psi^{2}}$.

The scaling regimes are associated with the fixed points (FPs) of the RG transformation. The asymptotic large scale behavior is governed by the infrared fixed points. Their coordinates can be found from the requirement that the $\beta$-functions vanish. The directed bond percolation process has only one $\beta$-function

$$
\beta_{u}=u\left(-2 \epsilon-\gamma_{u}\right) \approx u\left(-2 \epsilon+\frac{3 u}{4}-\frac{3 u \epsilon}{8}-\frac{3 u^{2} \epsilon}{128}-0.389626 u^{2}\right) .
$$

There are two FPs given by the equation above: the trivial (Gaussian or free) FP $(u=0)$ and the non-trivial one of the following form:

$$
u^{*}=\frac{8}{3} \epsilon+5.02756 \epsilon^{2}+O\left(\epsilon^{3}\right) .
$$

that corresponds to the critical percolation process. After the determination of the FP coordinates, critical exponents can be analyzed. First, the critical exponent $\eta$ takes the following value

$$
\left.\eta \equiv 2 \gamma_{\psi}\right|_{u=u^{*}}=-\frac{\epsilon}{3}-0.27228 \epsilon^{2}+O\left(\epsilon^{3}\right) .
$$

The second is the so-called dynamical exponent $z$ which is associated with the distinctive behavior with respect to the time direction

$$
z \equiv 2-\left.\gamma_{D}\right|_{u=u^{*}}=2-\frac{\epsilon}{6}-0.11682 \epsilon^{2}+O\left(\epsilon^{3}\right) .
$$

The needed momentum integrals have been calculated with a numerical precision of $10^{-4}$. For comparison with an analytic calculation, we report the appropriately changed results (rescaling $\epsilon \rightarrow 2 \epsilon$ is needed) from $[9,11]$ :

$$
\begin{aligned}
& \eta=-\frac{\epsilon}{3}\left[1+\left(\frac{25}{144}+\frac{161}{72} \ln \frac{4}{3}\right) \epsilon+O\left(\epsilon^{2}\right)\right] \approx-\frac{\epsilon}{3}-0.2723000633 \epsilon^{2}+O\left(\epsilon^{3}\right) \\
& z=2-\frac{\epsilon}{6}\left[1+\left(\frac{67}{144}+\frac{59}{72} \ln \frac{4}{3}\right) \epsilon+O\left(\epsilon^{2}\right)\right] \approx 2-\frac{\epsilon}{6}-0.1168362090 \epsilon^{2}+O\left(\epsilon^{3}\right)
\end{aligned}
$$


These values are in excellent agreement with our results (24) and (25).

Our two-loop results are in agreement with the analytic calculations and our numerical method is suitable for the calculation of the Feynman graphs to the three-loop order. To this end, it is necessary to take into account altogether 17 graphs for the one-irreducible Green function $\Gamma_{\psi^{\dagger} \psi}$ and 150 graphs for the function $\Gamma_{\psi^{\dagger} \psi^{2}}$. It is also feasible to use this method for higher-loop computations. One just has to keep in mind that in order to achieve the required accuracy, the computer time needed for the calculation of each of the diagrams is much longer. The work in this direction is in progress.

\section{Acknowledgements}

This work was supported by VEGA grant 1/0222/13. L. Ts. A. and M. V. K. acknowledge the Saint Petersburg State University for the research grant 11.38.185.2014. We would also like to thank Dr. M. Vala the coordinator of the project "Slovak infrastructure for high performance computing (SIVVP), ITMS 26230120002".

\section{References}

[1] A. N. Vasil'ev, The Field Theoretic Renormalization Group in Critical Behavior Theory and Stochastic Dynamics, [in Russian], PIYaF, St. Petersburg (1998); English trans., Chapman and Hall/CRC, Boca Raton, Fla (2004)

[2] D. J. Amit and V. Martín-Mayor, Field Theory, the Renormalization Group and Critical Phenomena, World Scientific, Singapore (2005)

[3] U. Täuber, Critical Dynamics: A Field Theory Approach to Equilibrium and Non-Equilibrium Scaling Behavior, Cambridge University Press, New York (2014)

[4] D. Stauffer and A. Aharony, Introduction to Percolation Theory, Taylor and Francis, London (1992)

[5] M. Henkel, H. Hinrichsen, and S. Lübeck Non-equilibrium phase transitions: Volume $1-A b$ sorbing phase transitions, Springer, Dordrecht (2004)

[6] F. Schlögl, Z. Phys. 253, 147 (1972)

[7] J. L. Cardy and R. L. Sugar, J. Phys. A 13, L423-L427 (1980)

[8] H. Hinrichsen, Adv. Phys. 49, 815-958 (2000)

[9] H. K. Janssen and U. C. Täuber, Ann. Phys. 315, 147-192 (2005)

[10] J. B. Bronzan, J. W. Dash, Phys. Rev. D 10, 4208; Phys. Rev. D 12, 1850 (1974)

[11] H. K. Janssen, Z. Phys. B: Condens. Matter 42, 151 (1981)

[12] L. Ts. Adzhemyan and M. V. Kompaniets, Theor. Math. Phys. 169, 1450-1459 (2011)

[13] L. Ts. Adzhemyan, M. V. Kompaniets, S. V. Novikov, and V. K. Sazonov, Theor. Math. Phys. 175 (3), 719-728 (2013)

[14] L. Ts. Adzhemyan and M. V. Kompaniets, Journal of Physics Conference Series 523, 012049 (2014)

[15] R. Kreckel, Comput. Phys. Commun. 106, 258-266 (1997); "Pvega.” ftp://ftphep.physik.unimainz.de/pub/pvegas/; "Cuba - a library for multidimensional numerical integration," http://feynarts.de/cuba/ 